Network Working Group

Request for Comments: 3143

I. Cooper

Category: Informational

\title{
Known HTTP Proxy/Caching Problems
}

\section{Status of this Memo}

This memo provides information for the Internet community. It does not specify an Internet standard of any kind. Distribution of this memo is unlimited.

Copyright Notice

Copyright (C) The Internet Society (2001). All Rights Reserved.

\section{Abstract}

This document catalogs a number of known problems with World Wide Web (WWW) (caching) proxies and cache servers. The goal of the document is to provide a discussion of the problems and proposed workarounds, and ultimately to improve conditions by illustrating problems. The construction of this document is a joint effort of the Web caching community.

Table of Contents

1. Introduction . . . . . . . . . . . . . . . . . . . . . . . 2

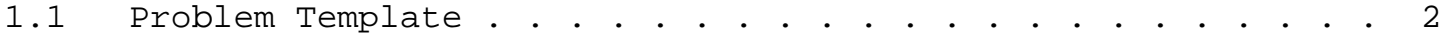

2. Known Problems. . . . . . . . . . . . . . . . . . . . . 4

2.1 Known Specification Problems . . . . . . . . . . . . . 5

2.1.1 Vary header is underspecified and/or misleading . . . . . 5

2.1.2 Client Chaining Loses Valuable Length Meta-Data . • . . . 9

2.2 Known Architectural Problems . . . . . . . . . . . . . . . 10

2.2.1 Interception proxies break client cache directives . . . . 10

2.2.2 Interception proxies prevent introduction of new HTTP

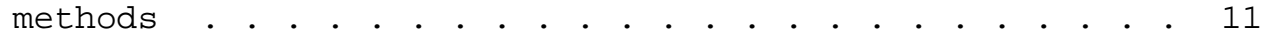

2.2.3 Interception proxies break IP address-based authentication . 12

2.2.4 Caching proxy peer selection in heterogeneous networks . . 13

2.2.5 ICP Performance . . . . . . . . . . . . . . . . . . 15

2.2.6 Caching proxy meshes can break HTTP serialization of content 16

2.3 Known Implementation Problems . . . . . . . . . . . . . . . 17

2.3.1 User agent/proxy failover . . . . . . . . . . . . . . . . 17

2.3.2 Some servers send bad Content-Length headers for files that contain $\mathrm{CR}$. . . . . . . . . . . . . . . . . 18 
3. Security Considerations . . . . . . . . . . . . . . . . . 18

References . . . . . . . . . . . . . . . . . . . 19

Authors' Addresses . . . . . . . . . . . . . . . 20

A. Archived Known Problems . . . . . . . . . . . . . . . . . . 21

A.1 Architectural . . . . . . . . . . . . . . . . . . . . . 21

A.1.1 Cannot specify multiple URIs for replicated resources . . 21

A.1.2 Replica distance is unknown . . . . . . . . . . . . . . . . 22

A.1.3 Proxy resource location . . . . . . . . . . . . . . . . 23

A.2 Implementation . . . . . . . . . . . . . . . . . . . . . . . 23

A.2.1 Use of Cache-Control headers . . . . . . . . . . . . . . . . 23

A.2.2 Lack of HTTP/1.1 compliance for caching proxies . . . . . 24

A.2.3 ETag support . . . . . . . . . . . . . . . . . . . . 25

A.2.4 Servers and content should be optimized for caching • • . 26

A.3 Administration . . . . . . . . . . . . . . . . . . 27

A.3.1 Lack of fine-grained, standardized hierarchy controls . . 27

A.3.2 Proxy/Server exhaustive log format standard for analysis . . 27

A.3.3 Trace log timestamps . . . . . . . . . . . . • . . . . . . 28

A.3.4 Exchange format for log summaries . . . . . . . . . . . . 29

Full Copyright Statement . . . . . . . . . . . . . . 32

1. Introduction

This memo discusses problems with proxies - which act as application-level intermediaries for Web requests - and more specifically with caching proxies, which retain copies of previously requested resources in the hope of improving overall quality of service by serving the content locally. Commonly used terminology in this memo can be found in the "Internet Web Replication and Caching Taxonomy" [2].

No individual or organization has complete knowledge of the known problems in Web caching, and the editors are grateful to the contributors to this document.

\subsection{Problem Template}

A common problem template is used within the following sections. We gratefully acknowledge RFC2525 [1] which helped define an initial format for this known problems list. The template format is summarized in the following table and described in more detail below.
Name:
short, descriptive name of the problem (3-5 words)
Classification:
Significance:
classifies the problem: performance, security, etc describes the problem succinctly
Implications:
See Also: magnitude of problem, environments where it exists the impact of the problem on systems and networks a reference to a related known problem
Indications: states how to detect the presence of this problem 


$\begin{array}{ll}\text { Solution(s): } & \text { describe the solution(s) to this problem, if any } \\ \text { Workaround: } & \text { practical workaround for the problem } \\ \text { References: } & \text { information about the problem or solution } \\ \text { Contact: } & \text { contact name and email address for this section }\end{array}$


Indications

How to detect the presence of the problem. This may include references to one or more substantiating documents that demonstrate the problem. This should include the network configuration that led to the problem such that it can be reproduced. Problems that are not reproducible will not appear in this memo.

Solution (s)

Solutions that permanently fix the problem, if such are known. For example, what version of the software does not exhibit the problem? Indicate if the solution is accepted by the community, one of several solutions pending agreement, or open possibly with experimental solutions.

Workaround

Practical workaround if no solution is available or usable. The workaround should have sufficient detail for someone experiencing the problem to get around it.

References

References to related information in technical publications or on the web. Where can someone interested in learning more go to find out more about this problem, its solution, or workarounds?

Contact

Contact name and email address of the person who supplied the information for this section. The editors are listed as contacts for anonymous submissions.

2. Known Problems

The remaining sections of this document present the currently documented known problems. The problems are ordered by classification and significance. Issues with protocol specification or architecture are first, followed by implementation issues. Issues of high significance are first, followed by lower significance.

Some of the problems initially identified in the previous versions of this document have been moved to Appendix A since they discuss issues where resolution primarily involves education rather than protocol work.

A full list of the problems is available in the table of contents. 


\subsection{Known Specification Problems}

2.1.1 Vary header is underspecified and/or misleading

Name

The "Vary" header is underspecified and/or misleading

Classification

Specification

Description

The Vary header in HTTP/1.1 was designed to allow a caching proxy to safely cache responses even if the server's choice of variants is not entirely understood. As RFC 2616 says:

The Vary header field can be used to express the parameters the server uses to select a representation that is subject to server-driven negotiation.

One might expect that this mechanism is useful in general for extensions that change the response message based on some aspects of the request. However, that is not true.

During the design of the HTTP delta encoding specification[9] it was realized that an HTTP/1.1 proxy that does not understand delta encoding might cache a delta-encoded response and then later deliver it to a non-delta-capable client, unless the extension included some mechanism to prevent this. Initially, it was thought that Vary would suffice, but the following scenario proves this wrong.

NOTE: It is likely that other scenarios exhibiting the same basic problem with "Vary" could be devised, without reference to delta encoding. This is simply a concrete scenario used to explain the problem.

A complete description of the IM and A-IM headers may be found in the "Delta encoding in HTTP" specification. For the purpose of this problem description, the relevant details are:

1. The concept of an "instance manipulation" is introduced. In some ways, this is similar to a content-coding, but there are differences. One example of an instance manipulation name is "vcdiff".

2. A client signals its willingness to accept one or more instance-manipulations using the A-IM header. 
3. A server indicates which instance-manipulations are used to encode the body of a response using the IM header.

4. Existing implementations will ignore the A-IM and IM headers, following the usual HTTP rules for handling unknown headers.

5. Responses encoded with an instance-manipulation are sent using the (proposed) 226 status code, "IM Used".

6. In response to a conditional request that carries an IM header, if the request-URI has been modified then a server may transmit a compact encoding of the modifications using a delta-encoding instead of a status-200 response. The encoded response cannot be understood by an implementation that does not support delta encodings.

This summary omits many details.

Suppose client A sends this request via proxy P:

GET http://example.com/foo.html HTTP/1.1

Host: example.com

If-None-Match: "abc"

A-IM: vcdiff

and the origin server returns, via $\mathrm{P}$, this response:

HTTP/1.1 226 IM Used

Etag: "def"

Date: Wed, 19 Apr 2000 18:46:13 GMT

IM: vcdiff

Cache-Control: $\max -$ age-60

Vary: A-IM, If-None-Match

the body of which is a delta-encoded response (it encodes the difference between the Etag "abc" instance of foo.html, and the "def" instance). Assume that $P$ stores this response in its cache, and that $P$ does not understand the vcdiff encoding.

Later, client B, also ignorant of delta-encoding, sends this request via $\mathrm{P}$ :

GET http://example.com/foo.html HTTP/1.1

Host: example.com

What can $P$ do now? According to the specification for the Vary header in RFC2616, 
The Vary field value indicates the set of request-header fields that fully determines, while the response is fresh, whether a cache is permitted to use the response to reply to a subsequent request without revalidation.

Implicitly, however, the cache would be allowed to use the stored response in response to client B WITH "revalidation". This is the potential bug.

An obvious implementation of the proxy would send this request to test whether its cache entry is fresh (i.e., to revalidate the entry) :

GET / foo.html HTTP/1.1

Host: example.com

If-None-Match: "def"

That is, the proxy simply forwards the new request, after doing the usual transformation on the URL and tacking on the "obvious" If-None-Match header.

If the origin server's Etag for the current instance is still "def", it would naturally respond:

HTTP/1.1 304 Not Modified

Etag: "def"

Date: Wed, 19 Apr 2000 18:46:14 GMT

thus telling the proxy $P$ that it can use its stored response. But this cache response actually involves a delta-encoding that would not be sensible to client B, signaled by a header field that would be ignored by $B$, and so the client displays garbage.

The problem here is that the original request (from client A) generated a response that is not sensible to client B, not merely one that is not "the appropriate representation" (as the result of server-driven negotiation).

One might argue that the proxy $\mathrm{P}$ shouldn't be storing status-226 responses in the first place. True in theory, perhaps, but unfortunately RFC2616, section 13.4, says:

A response received with any [status code other than 200, 203, $206,300,301$ or 410] MUST NOT be returned in a reply to a subsequent request unless there are cache-control directives or another header(s) that explicitly allow it. For example, these 
include the following: an Expires header (section 14.21); a

"max-age", "s-maxage", "must-revalidate", "proxy-revalidate", "public" or "private" cache-control directive (section 14.9).

In other words, the specification allows caching of responses with yet-to-be-defined status codes if the response carries a plausible Cache-Control directive. So unless we ban servers implementing this kind of extension from using these Cache-Control directives at all, the Vary header just won't work.

Significance Medium

Implications

Certain plausible extensions to the HTTP/1.1 protocol might not interoperate correctly with older HTTP/1.1 caches, if the extensions depend on an interpretation of Vary that is not the same as is used by the cache implementer.

This would have the effect either of causing hard-to-debug cache transparency failures, or of discouraging the deployment of such extensions, or of encouraging the implementers of such extensions to disable caching entirely.

Indications

The problem is visible when hand-simulating plausible message exchanges, especially when using the proposed delta encoding extension. It probably has not been visible in practice yet.

Solution (s)

1. Section 13.4 of the HTTP/1.1 specification should probably be changed to prohibit caching of responses with status codes that the cache doesn't understand, whether or not they include Expires headers and the like. (It might require some care to define what "understands" means, leaving room for future extensions with new status codes.) The behavior in this case needs to be defined as equivalent to "Cache-Control: no-store" rather than "no-cache", since the latter allows revalidation.

Possibly the specification of Vary should require that it be treated as "Cache-Control: no-store" whenever the status code is unknown - that should solve the problem in the scenario given here. 
2. Designers of HTTP/1.1 extensions should consider using mechanisms other than Vary to prevent false caching.

It is not clear whether the Vary mechanism is widely implemented in caches; if not, this favors solution \#1.

Workaround

A cache could treat the presence of a Vary header in a response as an implicit "Cache-control: no-store", except for "known" status codes, even though this is not required by RFC 2616. This would avoid any transparency failures. "Known status codes" for basic HTTP/1.1 caches probably include: 200, 203, 206, 300, 301, 410 (although this list should be re-evaluated in light of the problem discussed here).

References

See [9] for the specification of the delta encoding extension, as well as for an example of the use of a Cache-Control extension instead of "Vary."

Contact

Jeff Mogul <mogulepa.dec.com>

2.1.2 Client Chaining Loses Valuable Length Meta-Data

Name

Client Chaining Loses Valuable Length Meta-Data

Classification

Performance

Description

HTTP/1.1[3] implementations are prohibited from sending ContentLength headers with any message whose body has been TransferEncoded. Because 1.0 clients cannot accept chunked TransferEncodings, receiving 1.1 implementations must forward the body to 1.0 clients must do so without the benefit of information that was discarded earlier in the chain.

Significance

Low

Implications

Lacking either a chunked transfer encoding or Content-Length indication creates negative performance implications for how the proxy must forward the message body. 
In the case of response bodies, the server may either forward the response while closing the connection to indicate the end of the response or must utilize store and forward semantics to buffer the entire response in order to calculate a content-Length. The former option defeats the performance benefits of persistent connections in HTTP/1.1 (and their Keep-Alive cousin in HTTP/1.0) as well as creating some ambiguously lengthed responses. The latter store and forward option may not even be feasible given the size of the resource and it will always introduce increased latency.

Request bodies must undertake the store and forward process as 1.0 request bodies must be delimited by Content-Length headers. As with response bodies this may place unacceptable resource constraints on the proxy and the request may not be able to be satisfied.

Indications

The lack of HTTP/1.0 style persistent connections between 1.0 clients and 1.1 proxies, only when accessing 1.1 servers, is a strong indication of this problem.

Solution (s)

An HTTP specification clarification that would allow origin known identity document Content-Lengths to be carried end to end would alleviate this issue.

Workaround

None.

Contact

Patrick McManus <mcmanus@AppliedTheory.com>

\subsection{Known Architectural Problems}

2.2.1 Interception proxies break client cache directives

Name Interception proxies break client cache directives

Classification

Architecture

Description

HTTP[3] is designed for the user agent to be aware if it is connected to an origin server or to a proxy. User agents believing they are transacting with an origin server but which are 
really in a connection with an interception proxy may fail to send critical cache-control information they would have otherwise included in their request.

Significance

High

Implications

Clients may receive data that is not synchronized with the origin even when they request an end to end refresh, because of the lack of inclusion of either a "Cache-control: no-cache" or "mustrevalidate" header. These headers have no impact on origin server behavior so may not be included by the browser if it believes it is connected to that resource. Other related data implications are possible as well. For instance, data security may be compromised by the lack of inclusion of "private" or "no-store" clauses of the Cache-control header under similar conditions.

Indications

Easily detected by placing fresh (un-expired) content on a caching proxy while changing the authoritative copy, then requesting an end-to-end reload of the data through a proxy in both interception and explicit modes.

Solution (s)

Eliminate the need for interception proxies and IP spoofing, which will return correct context awareness to the client.

Workaround

Include relevant Cache-Control directives in every request at the cost of increased bandwidth and CPU requirements.

Contact

Patrick McManus <mcmanus@AppliedTheory.com>

2.2.2 Interception proxies prevent introduction of new HTTP methods

Name

Interception proxies prevent introduction of new HTTP methods

Classification

Architecture

Description

A proxy that receives a request with a method unknown to it is required to generate an HTTP 501 Error as a response. HTTP methods are designed to be extensible so there may be applications deployed with initial support just for the user agent and origin 
server. An interception proxy that hijacks requests which include new methods destined for servers that have implemented those methods creates a de-facto firewall where none may be intended.

Significance

Medium within interception proxy environments.

Implications

Renders new compliant applications useless unless modifications are made to proxy software. Because new methods are not required to be globally standardized it is impossible to keep up to date in the general case.

Solution (s)

Eliminate the need for interception proxies. A client receiving a 501 in a traditional HTTP environment may either choose to repeat the request to the origin server directly, or perhaps be configured to use a different proxy.

Workaround

Level 5 switches (sometimes called Level 7 or application layer switches) can be used to keep HTTP traffic with unknown methods out of the proxy. However, these devices have heavy buffering responsibilities, still require $\mathrm{TCP}$ sequence number spoofing, and do not interact well with persistent connections.

The HTTP/1.1 specification allows a proxy to switch over to tunnel mode when it receives a request with a method or HTTP version it does not understand how to handle.

Contact

Patrick McManus <mcmanuseAppliedTheory.com>

Henrik Nordstrom <hno@hem.passagen.se> (HTTP/1.1 clarification)

2.2.3 Interception proxies break IP address-based authentication

Name

Interception proxies break IP address-based authentication

Classification

Architecture

Description

Some web servers are not open for public access, but restrict themselves to accept only requests from certain IP address ranges for security reasons. Interception proxies alter the source (client) IP addresses to that of the proxy itself, without the 


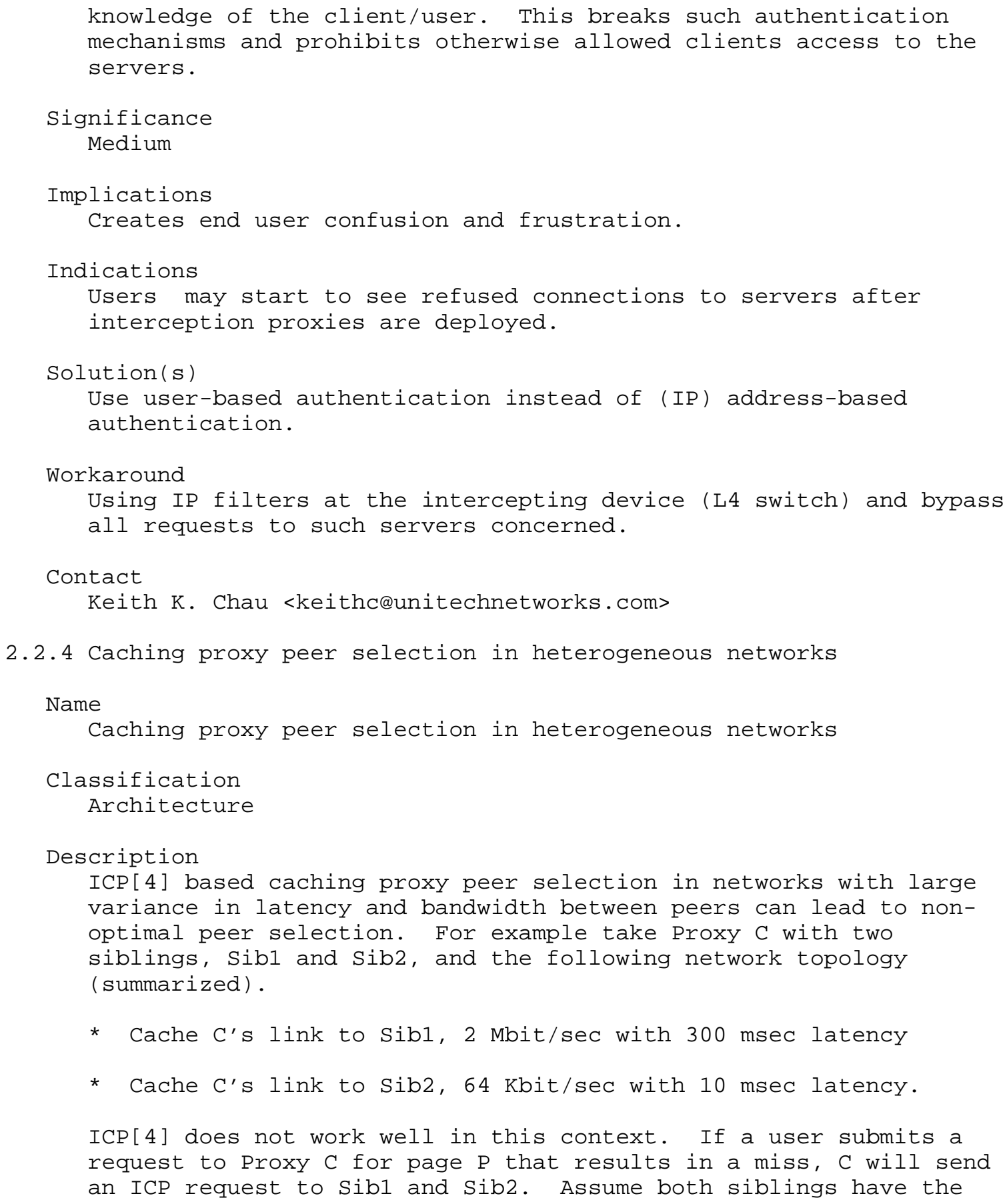


requested object $\mathrm{P}$. The ICP_HIT reply will always come from Sib2 before Sibl. However, it is clear that the retrieval of large objects will be faster from sib1, rather than sib2.

The problem is more complex because Sib1 and Sib2 can't have a $100 \%$ hit ratio. With a hit rate of $10 \%$, it is more efficient to use sibl with resources larger than $48 \mathrm{~K}$. The best choice depends on at least the hit rate and link characteristics; maybe other parameters as well.

Significance

Medium

Implications

By using the first peer to respond, peer selection algorithms are not optimizing retrieval latency to end users. Furthermore they are causing more work for the high-latency peer since it must respond to such requests but will never be chosen to serve content if the lower latency peer has a copy.

Indications

Inherent in design of ICP V1, ICP v2, and any cache mesh protocol that selects peers based upon first response.

This problem is not exhibited by cache digest or other protocols which (attempt to) maintain knowledge of peer contents and only hit peers that are believed to have a copy of the requested page.

Solution ( $\mathrm{s}$ )

This problem is architectural with the peer selection protocols.

Workaround

Cache mesh design when using such a protocol should be done in such a way that there is not a high latency variance among peers. In the example presented in the above description the high latency high bandwidth peer could be used as a parent, but should not be used as a sibling.

Contact

Ivan Lovric <ivan.lovricecnet.francetelecom.fr>

John Dilley <jad@akamai.com> 


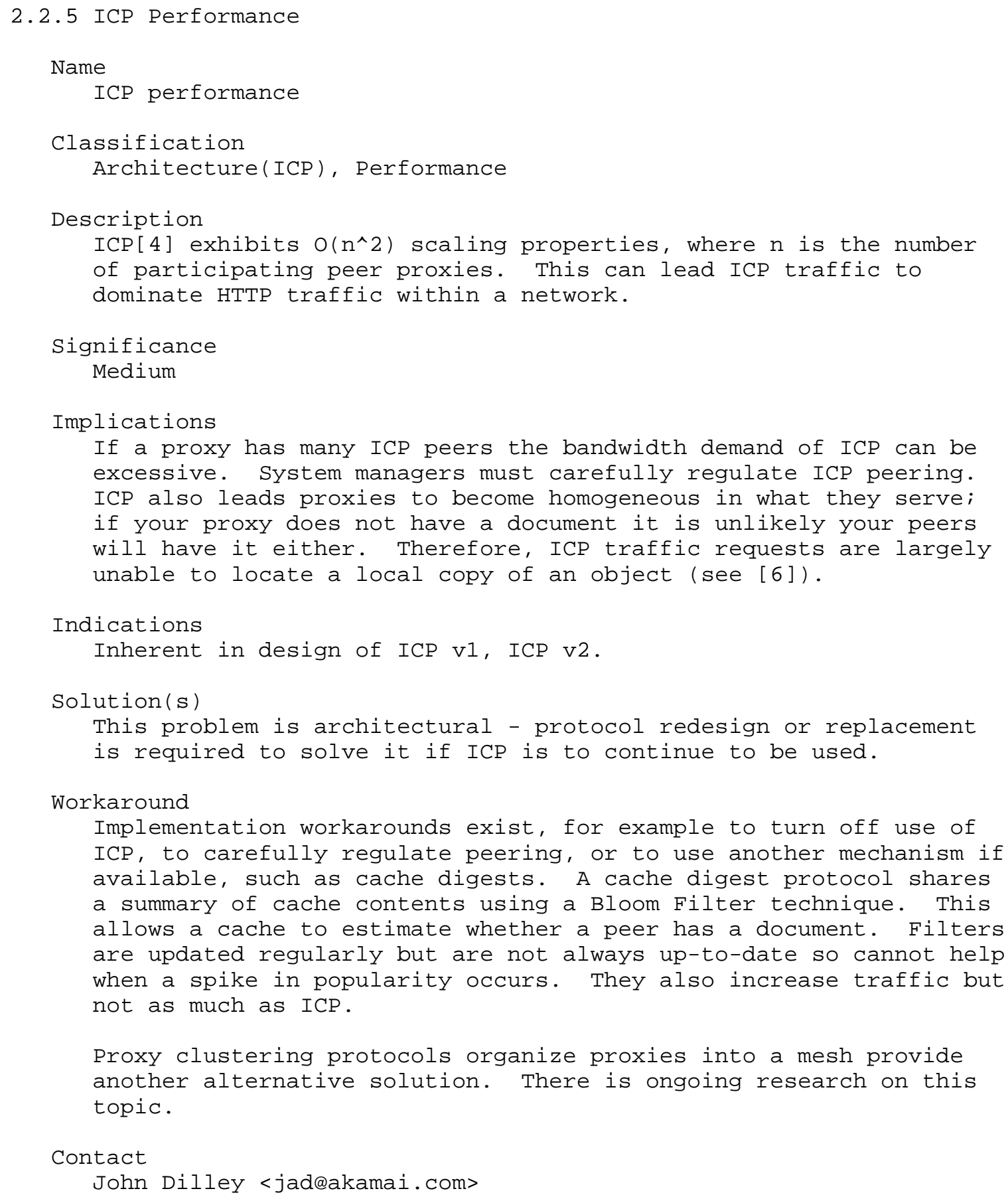




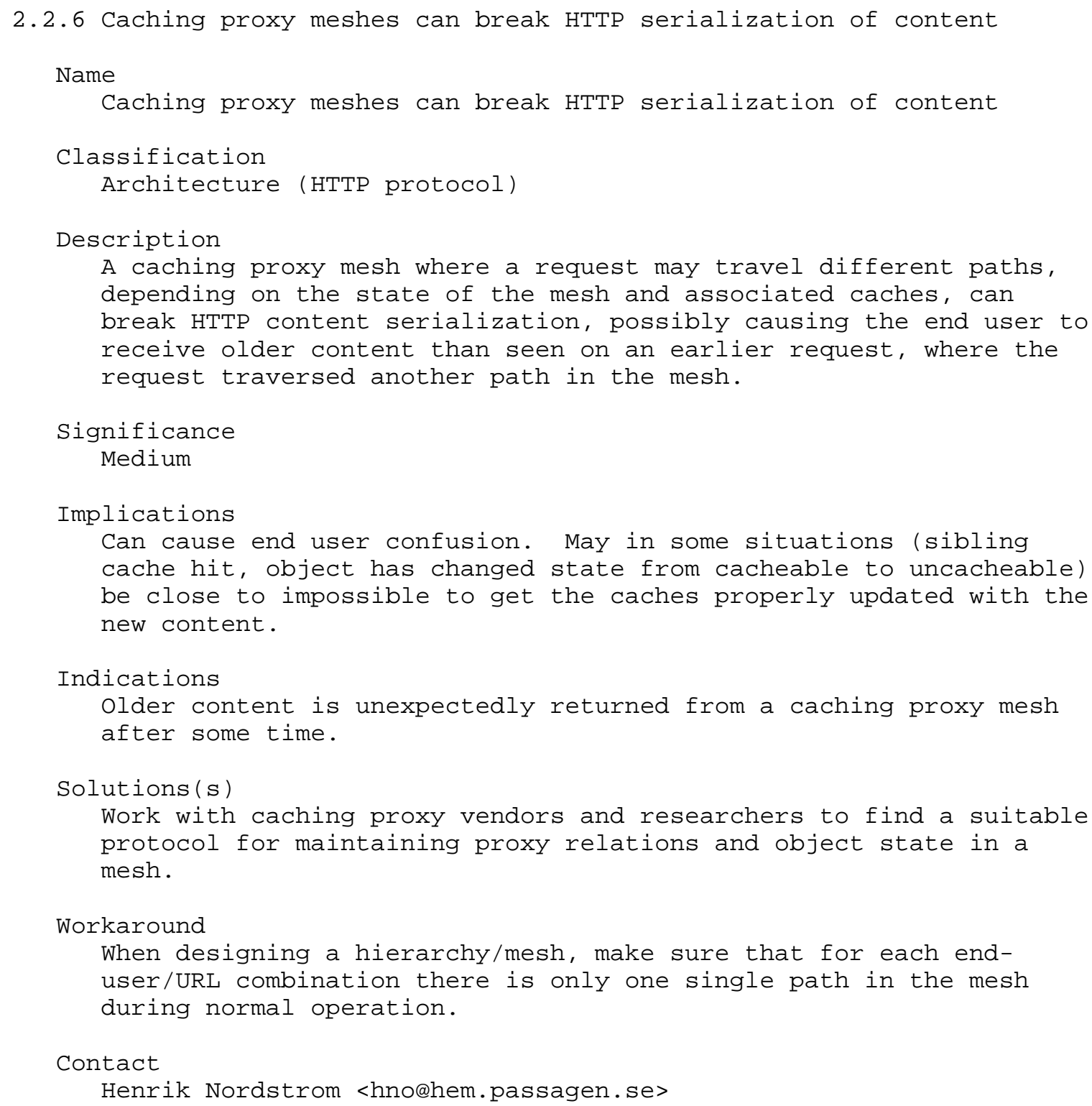




\subsection{Known Implementation Problems}

\subsubsection{User agent/proxy failover}

Name

User agent/proxy failover

Classification

Implementation

Description

Failover between proxies at the user agent (using a proxy.pac [8] file) is erratic and no standard behavior is defined. Additionally, behavior is hard-coded into the browser, so that proxy administrators cannot use failover at the user agent effectively.

Significance Medium

Implications Architects are forced to implement failover at the proxy itself, when it may be more appropriate and economical to do it within the user agent.

Indications

If a browser detects that its primary proxy is down, it will wait $\mathrm{n}$ minutes before trying the next one it is configured to use. It will then wait y minutes before asking the user if they'd like to try the original proxy again. This is very confusing for end users.

Solution (s)

Work with browser vendors to establish standard extensions to JavaScript proxy.pac libraries that will allow configuration of these timeouts.

Workaround User education; redundancy at the proxy level.

Contact

Mark Nottingham <mnot@mnot. net> 
2.3.2 Some servers send bad Content-Length headers for files that contain $\mathrm{CR}$

Name

Some servers send bad Content-Length headers for files that contain CR

Classification

Implementation

Description

Certain web servers send a Content-length value that is larger

than number of bytes in the HTTP message body. This happens when

the server strips off $C R$ characters from text files with lines terminated with CRLF as the file is written to the client. The server probably uses the stat() system call to get the file size for the Content-Length header. Servers that exhibit this behavior include the GN Web server (version 2.14 at least).

Significance Low. Surveys indicate only a small number of sites run faulty servers.

Implications

In this case, an HTTP client (e.g., user agent or proxy) may believe it received a partial response. HTTP/1.1 [3] advises that caches MAY store partial responses.

Indications

Count the number of bytes in the message body and compare to the Content-length value. If they differ the server exhibits this problem.

Solutions

Upgrade or replace the buggy server.

Workaround

Some browsers and proxies use one TCP connection per object and ignore the Content-Length. The document end of file is identified by the close of the TCP socket.

Contact

Duane Wessels <wessels@measurement-factory.com>

3. Security Considerations

This memo does not raise security considerations in itself. See the individual submissions for details of security concerns and issues. 
References

[1] Paxson, V., Allman, M., Dawson, S., Fenner, W., Griner, J., Heavens, I., Lahey, K., Semke, J. and B. Volz, "Known TCP Implementation Problems", RFC 2525, March 1999.

[2] Cooper, I., Melve, I. and G. Tomlinson, "Internet Web Replication and Caching Taxonomy", RFC 3040, January 2001.

[3] Fielding, R., Gettys, J., Mogul, J., Frystyk, H., Masinter, L., Leach, P. and T. Berners-Lee, "Hypertext Transfer Protocol -HTTP/1.1", RFC 2616, June 1999.

[4] Wessels, D. and K. Claffy, "Internet Cache Protocol (ICP), Version 2", RFC 2186, September 1997.

[5] Davison, B., "Web Traffic Logs: An Imperfect Resource for Evaluation", in Proceedings of the Ninth Annual Conference of the Internet Society (INET'99), July 1999.

[6] Melve, I., "Relation Analysis, Cache Meshes", in Proceedings of the 3rd International WWW Caching Workshop, June 1998, <http://wwwcache.ja.net/events/workshop/29/magicnumber.html>.

[7] Krishnamurthy, B. and M. Arlett, "PRO-COW: Protocol Compliance on the Web", AT\&T Labs Technical Report \#990803-05-TM, August 1999, <http://www.research.att.com/ bala/papers/procow-1.ps.gz>.

[8] Netscape, Inc., "Navigator Proxy Auto-Config File Format", March 1996 , http://home.netscape.com/eng/mozilla/2.0/relnotes/demo/proxylive.html

[9] Mogul, J., Krishnamurthy, B., Douglis, F., Feldmann, A., Goland, Y., van Hoff, A. and D. Hellerstein, "HTTP Delta in HTTP", Work in Progress. 


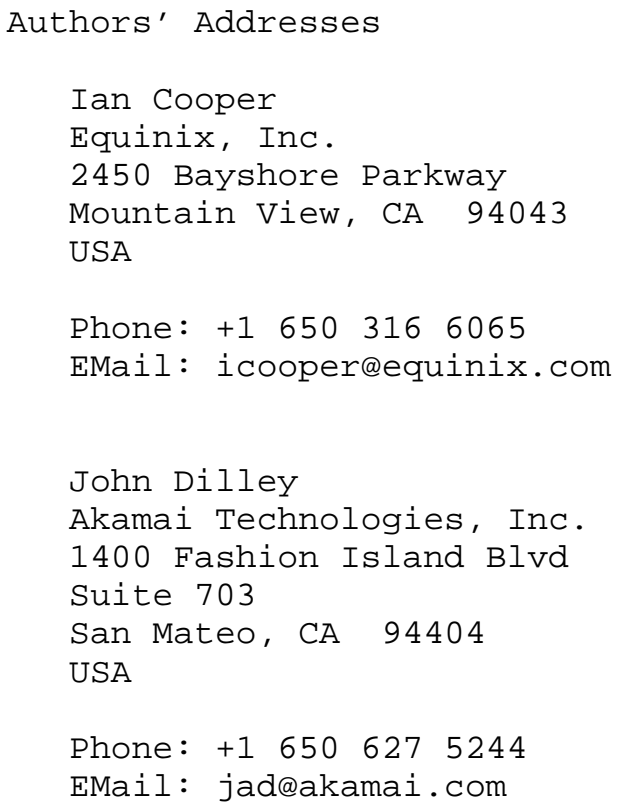


Appendix A. Archived Known Problems

The following sub-sections are an archive of problems identified in the initial production of this memo. These are typically problems requiring further work/research, or user education. They are included here for reference purposes only.

A. 1 Architectural

A.1.1 Cannot specify multiple URIs for replicated resources

Name

Cannot specify multiple URIs for replicated resources

Classification

Architecture

Description

There is no way to specify that multiple URIs may be used for a single resource, one for each replica of the resource. Similarly, there is no way to say that some set of proxies (each identified by a URI) may be used to resolve a URI.

Significance

Medium

Implications

Forces users to understand the replication model and mechanism. Makes it difficult to create a replication framework without protocol support for replication and naming.

Indications

Inherent in $\operatorname{HTTP} / 1.0, \operatorname{HTTP} / 1.1$.

Solution (s)

Architectural - protocol design is necessary.

Workaround

Replication mechanisms force users to locate a replica or mirror site for replicated content.

Contact

Daniel LaLiberte <liberte@w3.org> 


\section{A.1.2 Replica distance is unknown}

Name

Replica distance is unknown

Classification

Architecture

Description

There is no recommended way to find out which of several servers or proxies is closer either to the requesting client or to another machine, either geographically or in the network topology.

Significance Medium

Implications

Clients must guess which replica is closer to them when requesting a copy of a document that may be served from multiple locations. Users must know the set of servers that can serve a particular object. This in general is hard to determine and maintain. Users must understand network topology in order to choose the closest copy. Note that the closest copy is not always the one that will result in quickest service. A nearby but heavily loaded server may be slower than a more distant but lightly loaded server.

Indications

Inherent in $\operatorname{HTTP} / 1.0, \operatorname{HTTP} / 1.1$.

Solution (s)

Architectural - protocol work is necessary. This is a specific instance of a general problem in widely distributed systems. A general solution is unlikely, however a specific solution in the web context is possible.

Workaround

Servers can (many do) provide location hints in a replica selection web page. Users choose one based upon their location. Users can learn which replica server gives them best performance. Note that the closest replica geographically is not necessarily the closest in terms of network topology. Expecting users to understand network topology is unreasonable.

Contact

Daniel LaLiberte <liberteew3.org> 
A.1.3 Proxy resource location

Name

Proxy resource location

Classification

Architecture

Description

There is no way for a client or server (including another proxy) to inform a proxy of an alternate address (perhaps including the proxy to use to reach that address) to use to fetch a resource. If the client does not trust where the redirected resource came from, it may need to validate it or validate where it came from.

Significance

Medium

Implications

Proxies have no systematic way to locate resources within other proxies or origin servers. This makes it more difficult to share information among proxies. Information sharing would improve global efficiency.

Indications

Inherent in $\operatorname{HTTP} / 1.0, \operatorname{HTTP} / 1.1$.

Solution (s)

Architectural - protocol design is necessary.

Workaround

Certain proxies share location hints in the form of summary digests of their contents (e.g., Squid). Certain proxy protocols enable a proxy query another for its contents (e.g., ICP). (See however "ICP Performance" issue (Section 2.2.5).)

Contact

Daniel LaLiberte <libertedw3.org>

A.2 Implementation

A.2.1 Use of Cache-Control headers

Name

Use of Cache-Control headers

Classification

Implementation 


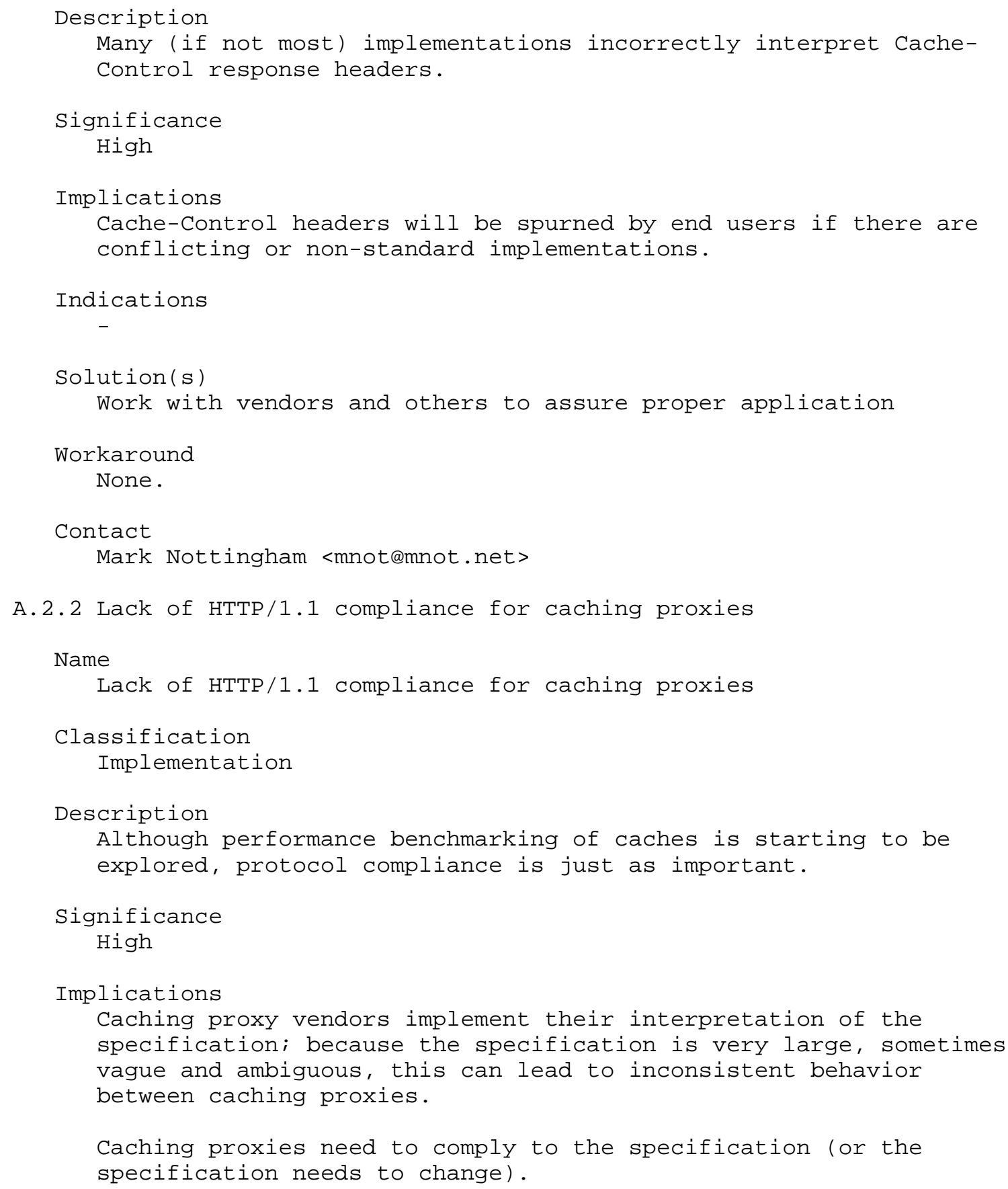




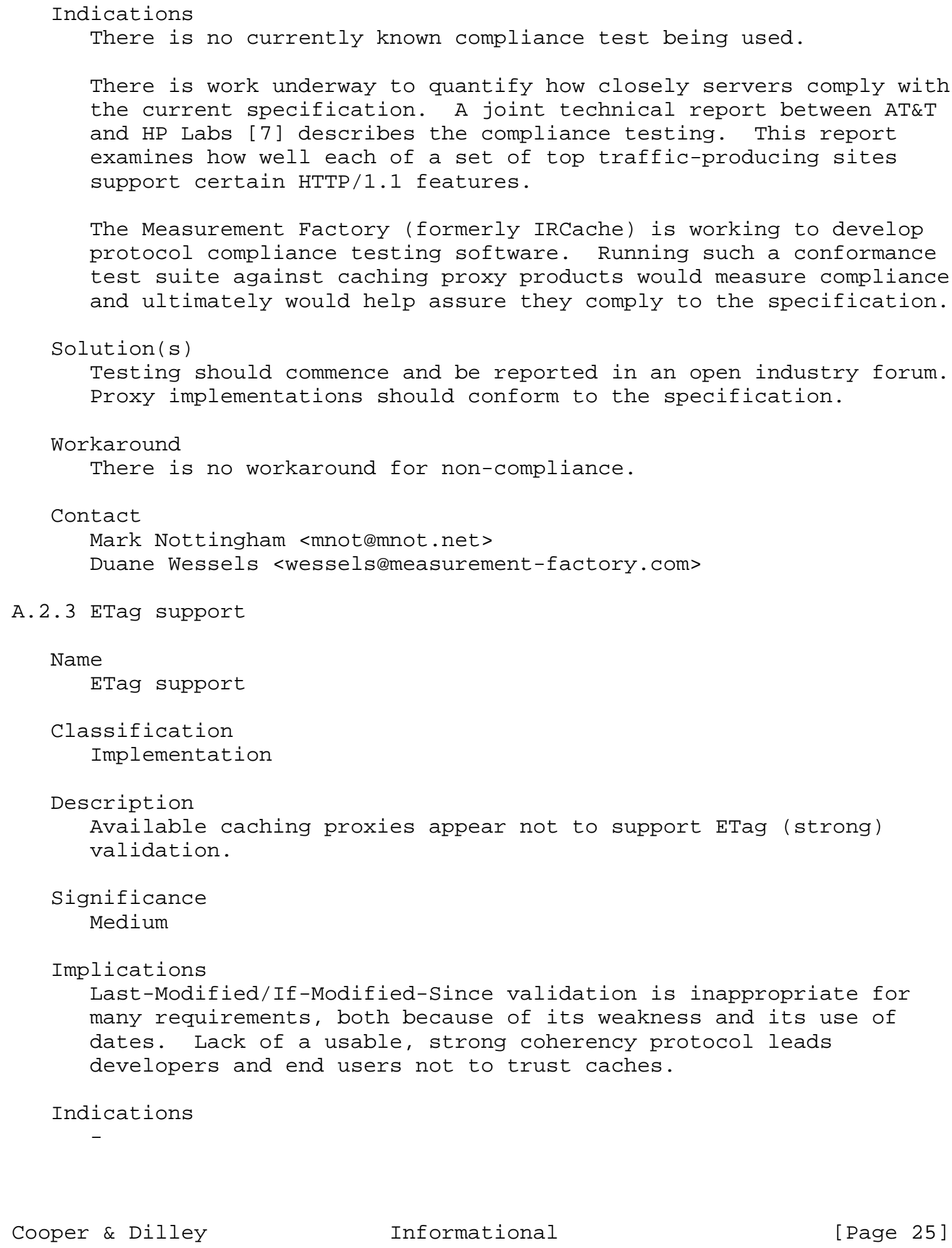




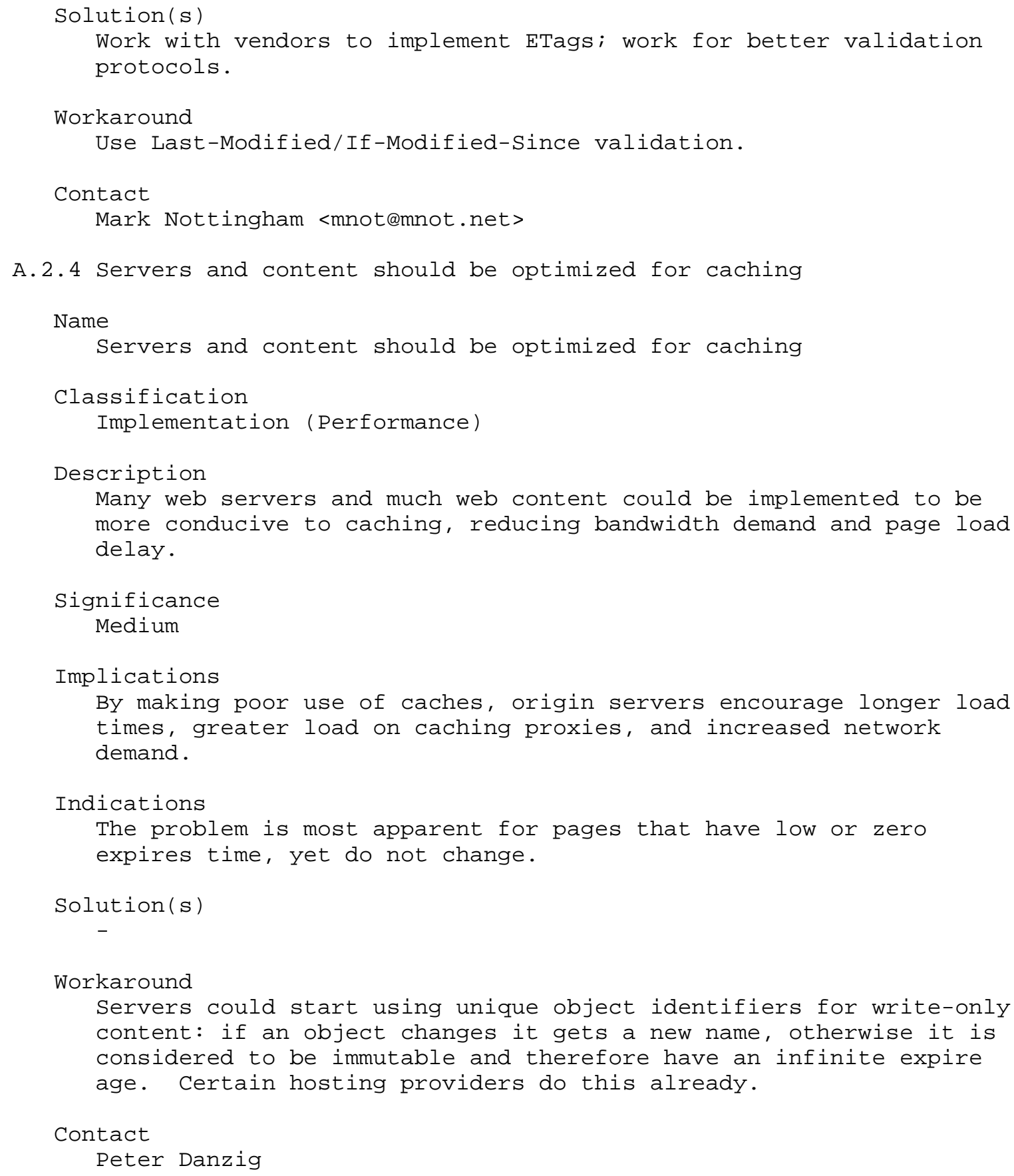




\section{A. 3 Administration}

A.3.1 Lack of fine-grained, standardized hierarchy controls

Name

Lack of fine-grained, standardized hierarchy controls

Classification

Administration

Description

There is no standard for instructing a proxy as to how it should resolve the parent to fetch a given object from. Implementations therefore vary greatly, and it can be difficult to make them interoperate correctly in a complex environment.

Significance Medium

Implications Complications in deployment of caches in a complex network (especially corporate networks)

Indications

Inability of some proxies to be configured to direct traffic based on domain name, reverse lookup IP address, raw IP address, in normal operation and in failover mode. Inability in some proxies to set a preferred parent / backup parent configuration.

Solution (s)

$-$

Workaround

Work with vendors to establish an acceptable configuration within the limits of their product; standardize on one product.

Contact

Mark Nottingham <mnot@mnot.net>

A.3.2 Proxy/Server exhaustive log format standard for analysis

Name

Proxy/Server exhaustive log format standard for analysis

Classification

Administration 


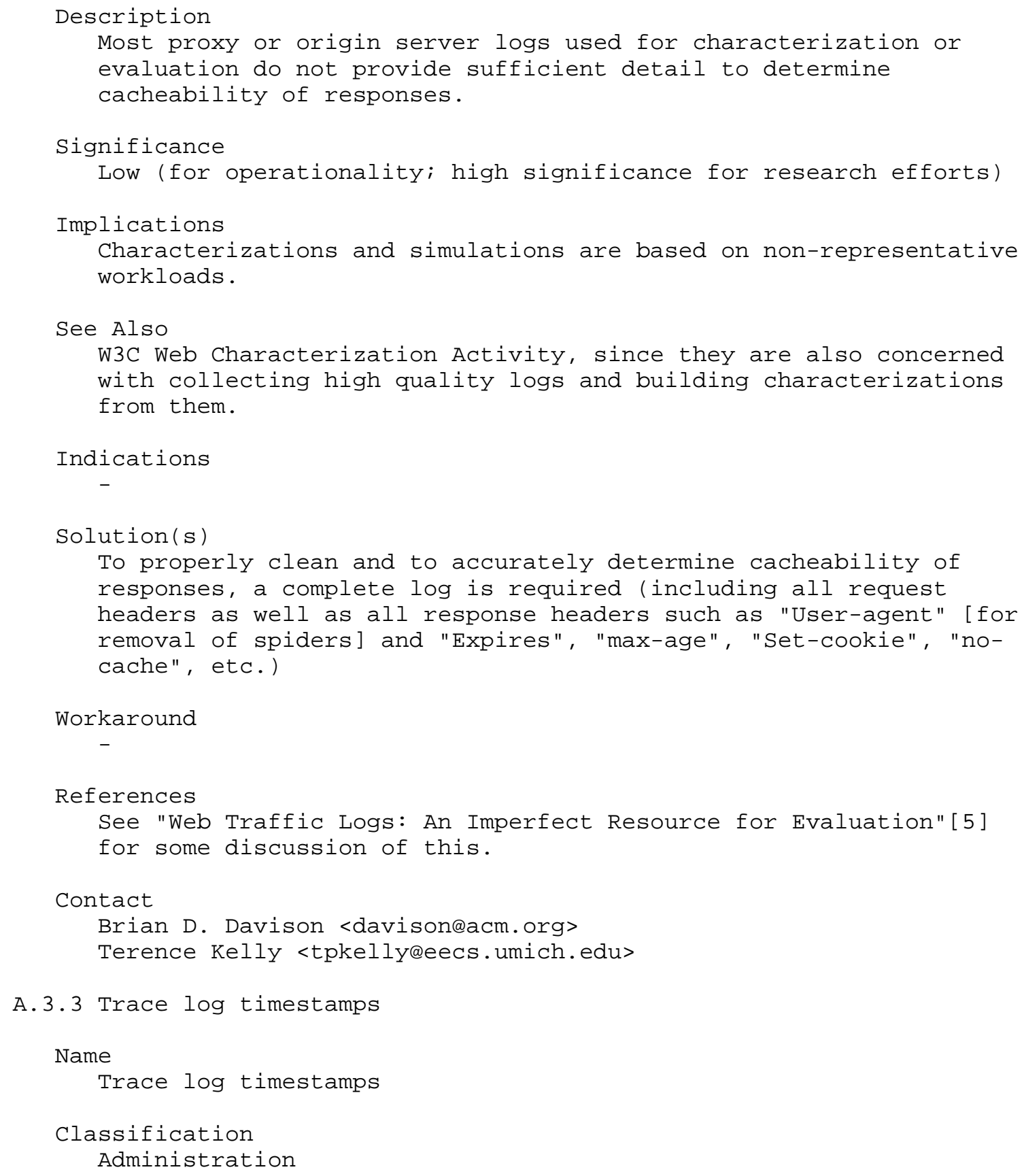




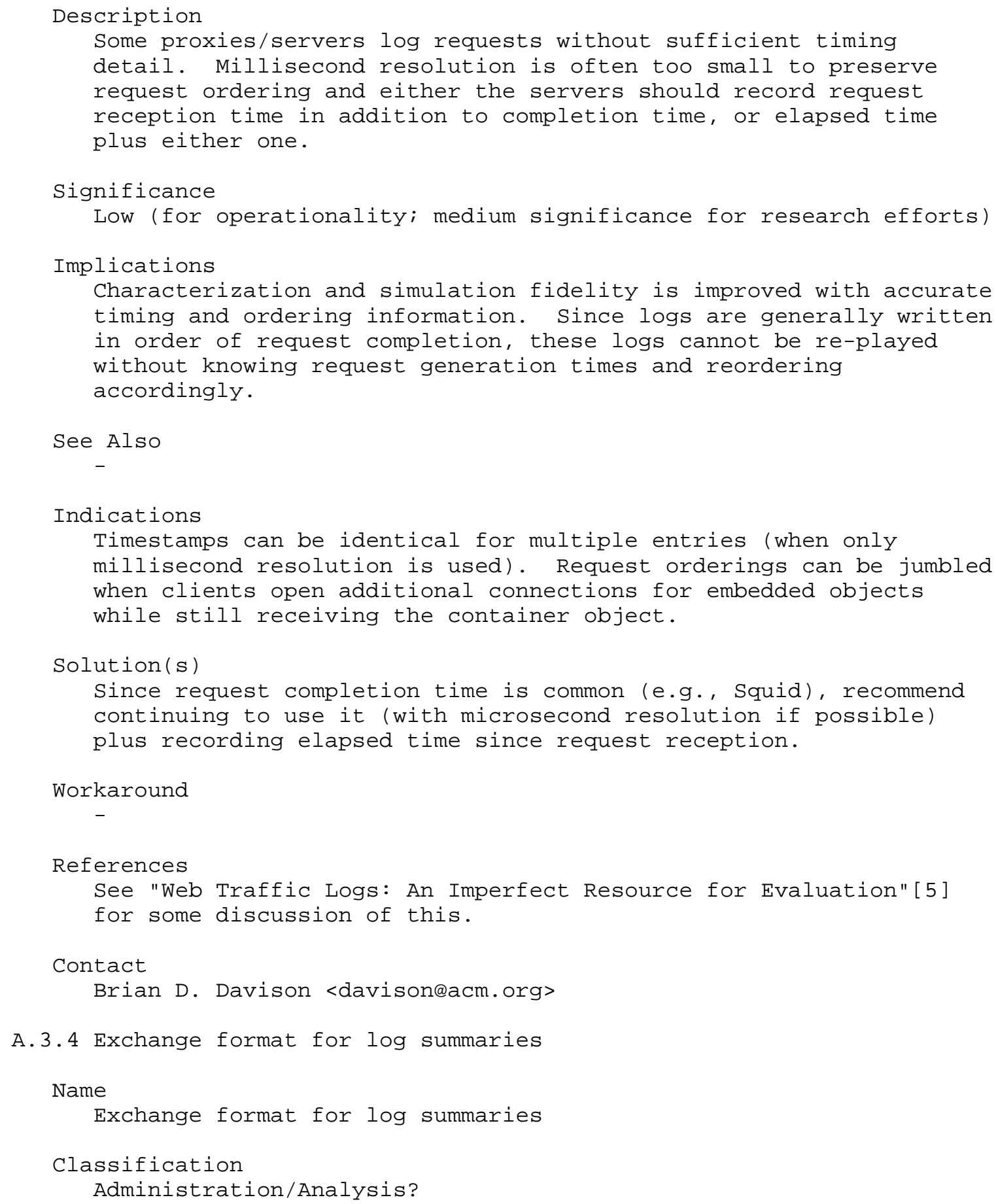


Description

Although we have (more or less) a standard log file format for proxies (plain vanilla Common Logfile and Squid), there isn't a commonly accepted format for summaries of those log files. Summaries could be generated by the cache itself, or by postprocessing existing log file formats such as squid's.

Significance

High, since it means that each log file summarizing/analysis tool is essentially reinventing the wheel (un-necessary repetition of code), and the cost of processing a large number of large log files through a variety of analysis tools is (again for no good reason) excessive.

Implications

In order to perform a meaningful analysis (e.g., to measure performance in relation to loading/configuration over time) the access logs from multiple busy caches, it's often necessary to run first one tool then another, each against the entire log file (or a significantly large subset of the log). With log files running into hundreds of MB even after compression (for a cache dealing with millions of transactions per dayl this is a non-trivial task.

See Also

IP packet/header sniffing - it may be that individual transactions are at a level of granularity which simply isn't sensible to be attempting on extremely busy caches. There may also be legal implications in some countries, e.g., if this analysis identifies individuals.

Indications

Disks/memory full(!) Stats (using multiple programs) take too long to run. Stats crunching must be distributed out to multiple machines because of its high computational cost.

Solution (s)

Have the proxy produce a standardized summary of its activity either automatically or via an external (e.g., third party) tool, in a commonly agreed format. The format could be something like XML or the Extended Common Logfile, but the format and contents are subjects for discussion. Ideally this approach would permit individual cache server products to supply subsets of the possible summary info, since it may not be feasible for all servers to provide all of the information which people would like to see. 


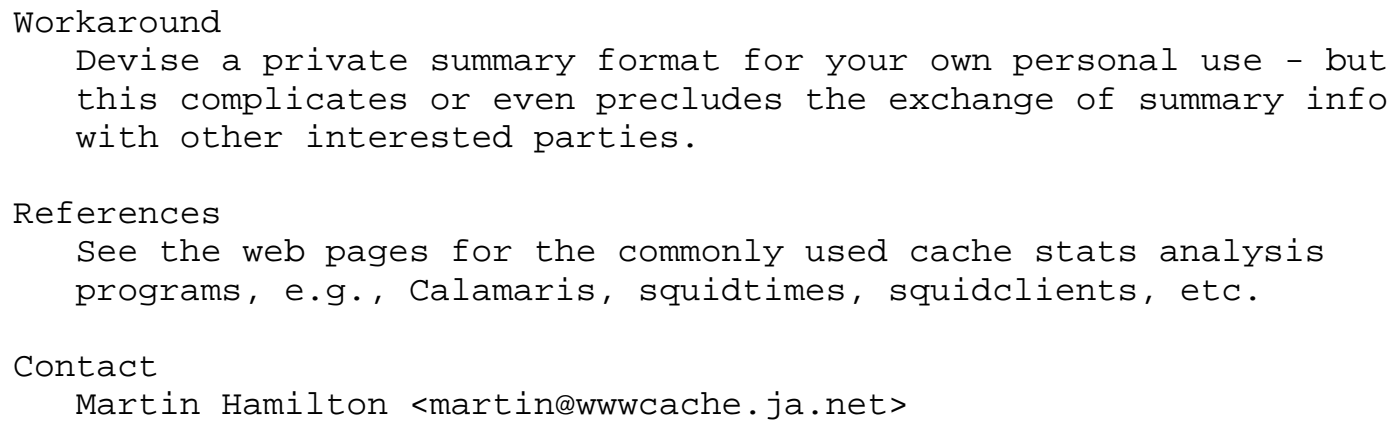


Full Copyright statement

Copyright (C) The Internet Society (2001). All Rights Reserved.

This document and translations of it may be copied and furnished to others, and derivative works that comment on or otherwise explain it or assist in its implementation may be prepared, copied, published and distributed, in whole or in part, without restriction of any kind, provided that the above copyright notice and this paragraph are included on all such copies and derivative works. However, this document itself may not be modified in any way, such as by removing the copyright notice or references to the Internet society or other Internet organizations, except as needed for the purpose of developing Internet standards in which case the procedures for copyrights defined in the Internet Standards process must be followed, or as required to translate it into languages other than English.

The limited permissions granted above are perpetual and will not be revoked by the Internet society or its successors or assigns.

This document and the information contained herein is provided on an "AS IS" basis and THE INTERNET SOCIETY AND THE INTERNET ENGINEERING TASK FORCE DISCLAIMS ALL WARRANTIES, EXPRESS OR IMPLIED, INCLUDING BUT NOT LIMITED TO ANY WARRANTY THAT THE USE OF THE INFORMATION HEREIN WILL NOT INFRINGE ANY RIGHTS OR ANY IMPLIED WARRANTIES OF MERCHANTABILITY OR FITNESS FOR A PARTICULAR PURPOSE.

Acknowledgement

Funding for the RFC Editor function is currently provided by the Internet Society. 